\title{
Fluorescent Biocompatible Tactic PMMA-QDs Thin Films
}

\author{
Cashe LESTER ${ }^{1}$, Abdulsalam ABDULKADIR ${ }^{1}$, Lauren WASHBURN ${ }^{1}$, \\ Ashwinin KUCKNOOR ${ }^{2}$, Su-Ying WEl ${ }^{1, a, *}$ \\ ${ }^{1}$ Department of Chemistry and Biochemistry, Lamar University, Beaumont, TX 77710, USA \\ ${ }^{2}$ Department of Biology, Lamar University, Beaumont, TX 77710, USA \\ asuying.wei@lamar.edu \\ ${ }^{*}$ Corresponding author
}

Keywords: Fluorescent, Biocompatible, Tactic PMMA, QDs.

\begin{abstract}
Poly(methyl methacrylate) thin films were prepared through solvent casting method followed by thermal treatment to completely remove the residual solvent. All three different tactic PMMAs were studied; the PMMA solution was made in chloroform at various concentrations, with $0.1 \mathrm{wt} \%$ QDs added against the polymer mass in the polymer solution. PMMA-QDs thin films as well as the pristine PMMA thin film (as control sample) were characterized using a variety of analytical techniques to probe their structure, functionalities, and respective physicochemical properties including thickness, surface wettability, fluorescence emission, and biocompatibility using HeLa mammalian cell as a model cell line. The fluorescence emission steadily increased when the concentration of PMMA increases. The wettability test showed i-PMMA to be more hydrophilic with a smaller contact angle than the other tacticity, while a-PMMA showed to be more hydrophobic with a larger contact angle than the other tacticity. All PMMA-QDs thin films used in co-culturing with HeLa cells supported their growth and proliferation.
\end{abstract}

\section{Introduction}

Poly (methyl methacrylate), PMMA, is a broadly studied and well applied material. It is a tough, highly transparent material with excellent resistance to ultraviolet radiation and weathering. These properties make it ideal for many applications including biomedical, architecture and construction, and electronics. It can be colored, molded, cut, drilled, and formed; these properties allow for PMMA to be molded and formed to different types of products. The biocompatibility, high reliability, ease of manipulation of PMMA makes it a perfect material for dental applications such as cavity fillings. PMMA is also used in making incubators because it is safe, easy to clean, and durable. In addition, PMMA has been used for manufacturing medical equipment such as blood cuvette and drug testing device as well as food packaging products. In this project, PMMA properties were studied when the material was made as a thin film. Thin films polymers have many applications in industrial and biomedical fields. The study of thin films is increasing to meet the demands in the miniaturization of devices, including transistors, sensors, photodetectors, LEDs, and nonvolatile memory devices.[1]

The tacticity of a polymer comes from the way substituents are arranged on the polymer backbone in relation to the stereochemistry of adjacent chiral center. There are three different tacticities, namely atactic, syndiotactic, and isotactic. Atactic is referring to the polymer with the substituents arranged in an irregular fashion; for isotactic, the substituents are all on the same side of the chain; for syndiotactic, the substituents alternate regular from one side of the chain to the other. Figure 1 shows the stereoregular structures of the three tactic PMMAs. 


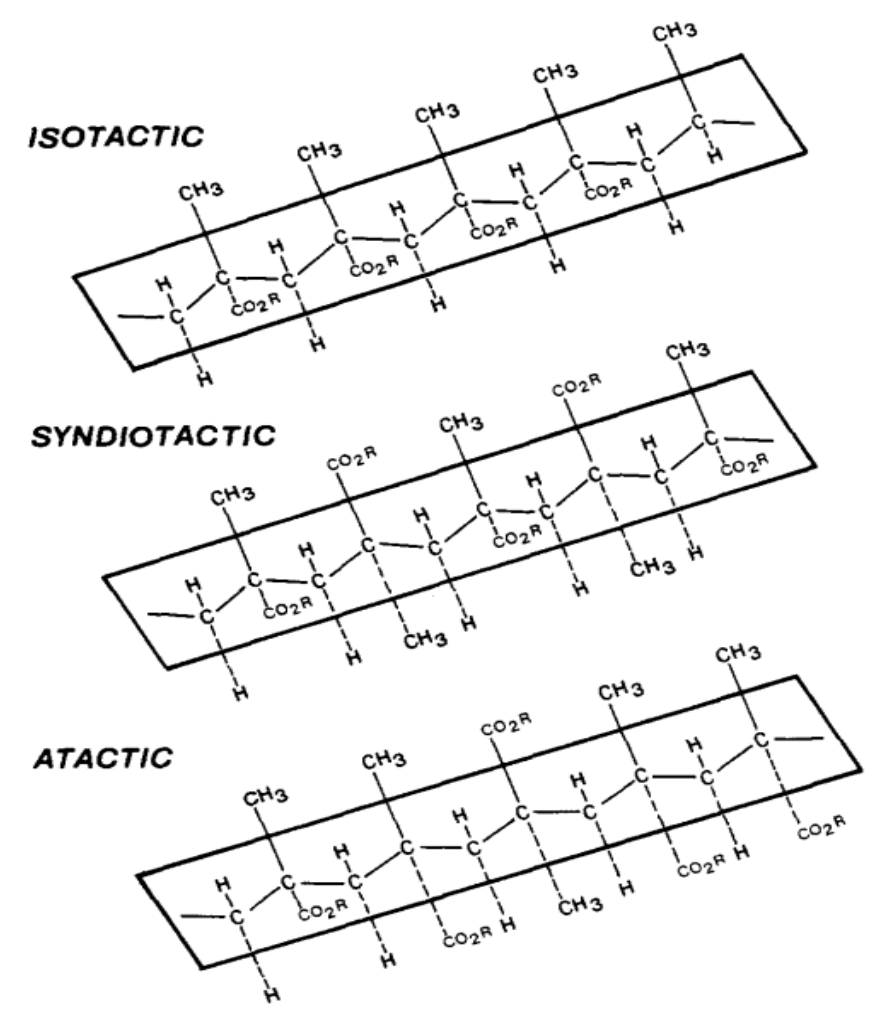

Fig. 1 The three different types of tacticities for poly (methyl methacrylate). Reprinted with permission from R. O. Loutfy, D. M. Teegarden, Effect of polymer chain tacticity on the fluorescence of molecular rotors, Macromolecules 16 (1983) 452- 456. Copyright 1983 American Chemical Society [2]

Fluorescent materials have been in high demand during the past few decades because of their important application in the field of materials and life science [3]. Owing to its excellent optical and mechanical properties, PMMA has been a first-choice component for the preparation of inorganic-organic composites.[1, 4-5] Quantum dots (QDs) are semiconductor nanocrystals with bright, stable and size-tunable photoluminescence (PL). Incorporation of QDs at high densities in polymer thin films is promising for the development of nanoscale sensors, light-emitting diodes, and photovoltaic devices.[6] In this paper, QDs were incorporated into all three different tactic PMMAs during the thin film preparation process, followed with detailed studies on the structure, fluorescence emission, wettability, and biocompatibility using HeLa cell as the model cell line.

\section{Experimental}

\section{Materials}

Three poly(methyl methacrylate), PMMA, polymers were used for this study; atactic PMMA (a-PMMA) with a molecular weight of 540,000 $/ \mathrm{mol}$, isotactic PMMA (i-PMMA; $98 \%$ isotactic) with a molecular weight of 300,000 $/ \mathrm{mol}$, and syndiotactic PMMA (s-PMMA; 85\% syndiotactic) with a molecular weight of 50,000 g/mol, were purchased from Scientific Polymer Products (Ontario, NY, USA) and used without any further treatment. The relative density for all three PMMAs is the same at 1.20. Chloroform (99\% and HPLC grade) was purchased from EM Science. Core-shell $\mathrm{CdSe} / \mathrm{ZnS}$ quantum dot (QDs; QSO-620-200) was a gift from Ocean Nanotech LLC at a concentration of $13.33 \mathrm{mg} / \mathrm{mL}$ in chloroform.

\section{Preparation of PMMA and PMMA-QDs Thin Films}

Each of the PMMA and PMMA/QDs solutions was prepared in a glass vial at certain concentrations in the chloroform, and mixed thoroughly by vortexing (Vortex genie 2; Scientific 
Industries). Then 5-mL of the solution was poured into a glass petri dish with lid and let it evaporate at room temperature. The wet film was further dried by placing in a vacuum oven at high temperature to completely remove the solvent. When the film was cooled to room temperature in the atmosphere, it was taken out by scouring the edge of the petri dish and floating in distilled water. The film was taken out and blotted dry with Kim wipe tissue for further characterization and property analysis. In summary, for the pristine PMMA thin films, the weight percentage of 1.0, 2.0, 3.0 and 5.0wt\% were prepared, while for the PMMA/QDs thin films, the weight percentage of the polymer in the solvent was remained the same as the pristine PMMAs while $0.1 \mathrm{wt} \%$ QDs (against the polymer) was added.

\section{Characterization and Property Analysis of All the Films}

The thickness of the thin films was measured with a device called Mitutoyo Absolute (Model No.C112EXB). At least five representative spots on the film were measured and the average and standard deviation were calculated and reported. The surface functionality analysis of all the PMMA and PMMA/QDs was obtained by attenuated total reflectance Fourier transform infrared spectroscopy (ATR-FTIR; Bruker Alpha), with a scan range of $4000-500 \mathrm{~cm}^{-1}$ at 32 scans. The fluorescence emission of all the different PMMA-QDs thin films was measured with a fluorescence spectrometer (Jasco Spectrometer Model Number FP8300). Surface wettability and surface energy of all thin films was determined by contact angle measurement using a goniometer (Future Digital Corp. Model OCA20); at least seven representative spots were measured and average and standard deviation were reported. For the cell biocompatibility study, HeLa cells were cultured in Dulbecco's Modified Eagle Media (DMEM) containing 10\% fetal bovine serum (FBS) and $100 \mathrm{u} / \mathrm{mL}$ penicillin-streptomycin, under standard conditions of $37{ }^{\circ} \mathrm{C}$ in a tissue culture incubator with $5 \% \mathrm{CO}_{2}$. No growth promoting agents were added to this medium. Cell trypsinization was then conducted to suspend the cells and prevent the cell from adhering to the petri dish for easy of enumeration by using a hemocytometer. The thin films were first sterilized in $70 \%$ ethanol by soaking them for about $30 \mathrm{~min}$ in a petri dish, and then exposed to UV light for an hour in a laminar air flow hood in order to maintain aseptic conditions. The thin films were then wetted with plain DMEM and air dried before placing onto wells on 8-well chambered slides. Two controlled sample was also prepared with only a cell culture and no films, each well containing the equal amount of films and $1 \times 10^{4}$ cells and the slide were incubated for 48 hours. After a period of incubation, the culture media was pipetted out of the well and $200 \mu \mathrm{L}$ of $1 \mathrm{x}$ phosphate buffered saline (PBS) was added to each well. Cell viability assay was then done by staining the cells with methylene blue dye for $10 \mathrm{~min}$. The excess stain was washed off with 1x PBS buffer and the co-culture films were transferred to new glass slides and viewed under BX41 Olympus microscope, and the image captured by using Cellsens software.

\section{Results and Discussion}

\section{IR Analysis of the Three Types of As-received PMMA}

Figure 2 is the ATR-FTIR spectra of the as-received a-PMMA, i-PMMA, and s-PMMA. The peaks observed at $2998 \mathrm{~cm}^{-1}$ and $2949 \mathrm{~cm}^{-1}$ are caused by the $\mathrm{SP}^{3} \mathrm{C}-\mathrm{H}$ stretching vibrations and a carbonyl stretching peak appears at $1720 \mathrm{~cm}^{-1}$. The bending vibrational mode of $\mathrm{H}-\mathrm{C}-\mathrm{H}$ shows at $1442 \mathrm{~cm}^{-1}$ and $1383 \mathrm{~cm}^{-1}$. A deformation peak at $1478 \mathrm{~cm}^{-1}$ corresponds to $\mathrm{C}-\mathrm{H}$ stretching vibration in $\mathrm{CH}_{3}$ while $\mathrm{C}-\mathrm{C}$ stretching appears at 989 and $949 \mathrm{~cm}^{-1}$. In the fingerprint region, the $\mathrm{C}=\mathrm{O}$ in plane bending shows at $810 \mathrm{~cm}^{-1}$ and the out of plane bending shows at $756 \mathrm{~cm}^{-1}$. Additional peak at $700 \mathrm{~cm}^{-1}$ is corresponding to the $\mathrm{C}-\mathrm{H}$ bending in the $\mathrm{CH}_{2}$ group of the PMMA backbone.

It has been proven that peaks in the region of $1300-1100 \mathrm{~cm}^{-1}$ are sensitive to conformational changes. $^{31,32}$ The other regions affected by conformational changes include $1750-1700 \mathrm{~cm}^{-1}$ and 1000- $700 \mathrm{~cm}^{-1}$ corresponding to $\mathrm{C}=\mathrm{O}$ stretching. While a-PMMA and s-PMMA spectra are similar, i-PMMA has a couple of distinct peaks. The peak at $1375 \mathrm{~cm}^{-1}$ for i-PMMA is very sharp compared to that in s-PMMA and a-PMMA. Both s-PMMA and a-PMMA have a doublet around $1236 \mathrm{~cm}^{-1}$ while 
i-PMMA shows a singlet. For the peak at $750 \mathrm{~cm}^{-1}$, i-PMMA has a doublet while s-PMMA has a single sharp peak and a-PMMA has a broad small peak in this region.

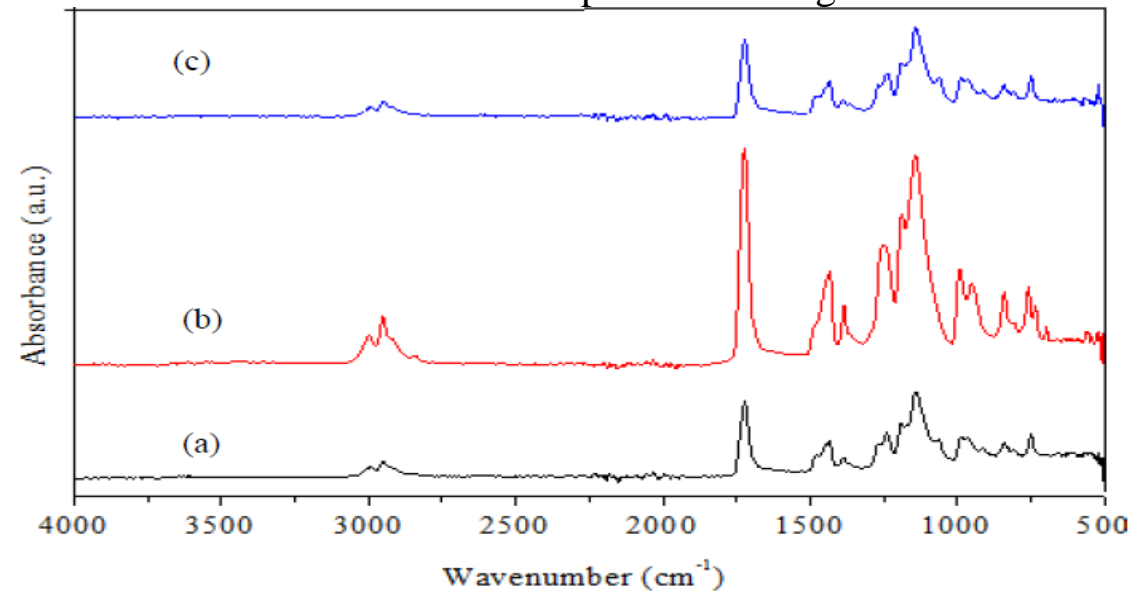

Fig. 2 IR spectra of as-received (a) a-PMMA, (b) i-PMMA, and (c) s-PMMA

\section{Fluorescence Emission Analysis of the Various PMMA-QDs Thin Films at Different Thickness}

The fluorescence emission was measured by passing a single wavelength through the PMMA-QDs thin films. The emission was collected after the absorption at the excited wavelength, and re-emission of the energy at longer wavelengths due to electronic transitions. The films have a fluorescence emission maximum wavelength at $614 \mathrm{~nm}$, which is the same as that of the as-received QDs solution in chloroform. Neat PMMAs do not absorb in the visible region and also contains no fluorophores, therefore there is no fluorescence emission for any neat PMMA films.

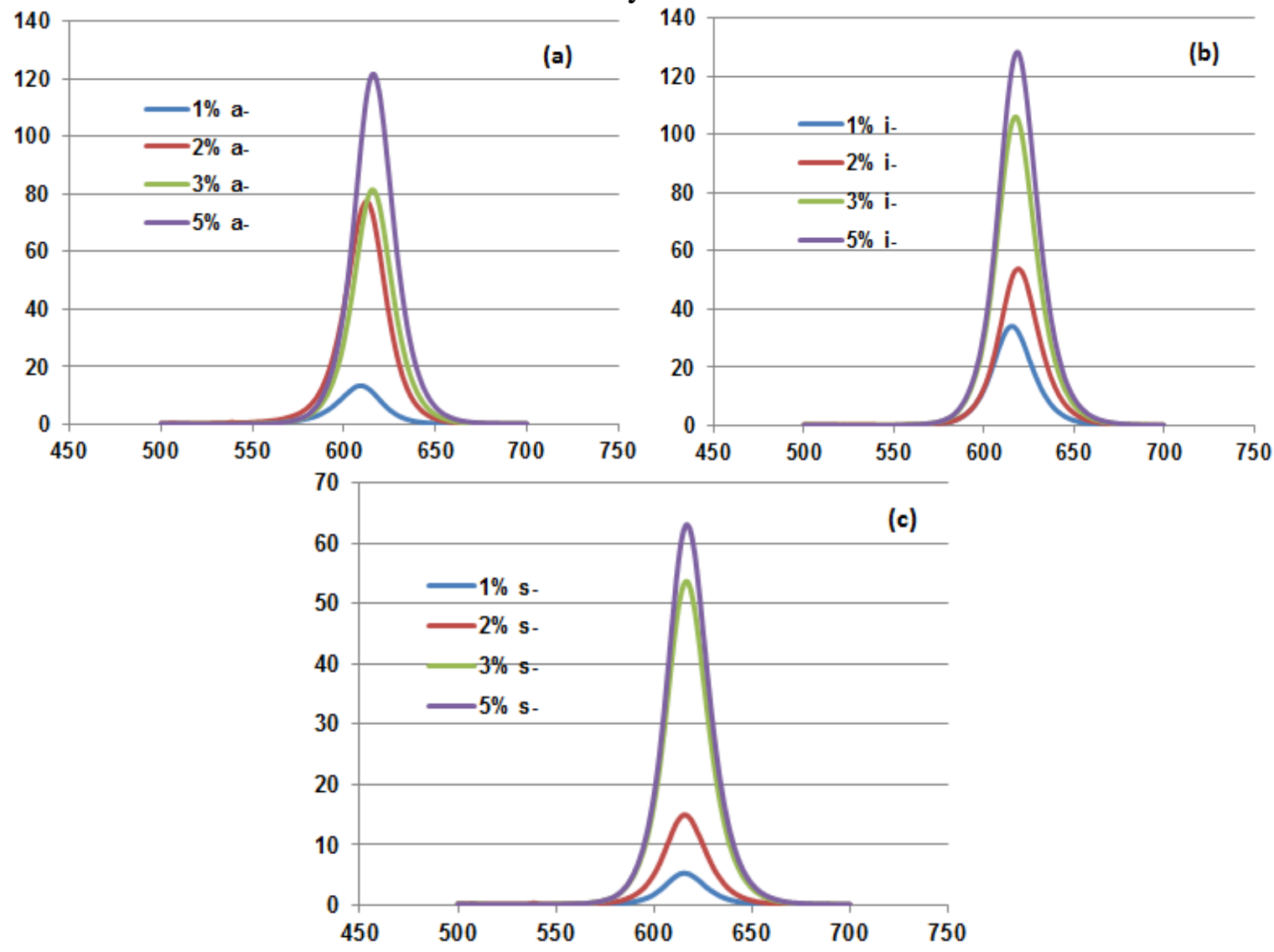

Fig. 3 Fluorescence analysis of the PMMA-QDs nanocomposite thin films

Figure 3a-c shows the fluorescence emission spectra of each tactic PMMA + 0.1wt\% QDs thin films at various concentration, 1.0, 2.0, 3.0, 5.0wt \%, respectively. It is observed that the fluorescence 
emission is increased with the increasing concentration for three PMMA-QDs thin films, which is due to the fact that the thickness of the thin film is increasing with increasing concentration, while the mass percentage of the QDs (against the PMMA) is fixed at $0.1 \mathrm{wt} \%$.

\section{Contact Angle of Different Tactic PMMA Surfaces}

The contact angle is a measure of hydrophilicity (wettability) of a solid surface using a liquid. Typically distilled water is used as the liquid. Factors to consider when measuring the contact angle are the intrinsic viscosity and Barry number of the liquid [7, 8].The relative strength of a liquid, solid and vapor molecular interaction is a function of its contact angle, if the contact angle is small then the thin film is said to be hydrophilic, and hydrophobic when it is high. Figure 4a-c displays the images, where a thin film, water droplet and vapor are at equilibrium for 5wt $\%$ a-PMMA, i-PMMA and s-PMMA.

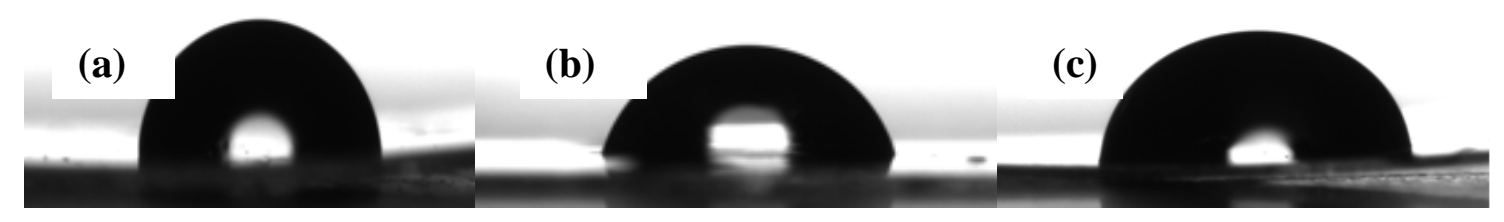

Fig. 4 Water droplet on 5wt\% of (a) a-PMMA, (b) i-PMMA, and (c) s-PMMA film. The corresponding contact angle is $90.25 \pm 5.75,74.59 \pm 3.28,89.11 \pm 4.45$ respectively

In summary, i-PMMA has the smallest contact angle for both neat and i-PMMA-QDs thin films, which indicates that i-PMMA films are more hydrophilic than the other tacticity. a-PMMA and s-PMMA are similar in terms of hydrophilicity, as the a-PMMA is syndiotactic rich polymer.

\section{HeLa Cell Biocompatibility Studies}

Cell proliferation assays show that PMMA and its QDs nanocomposite thin films used for co-culturing with HeLa cells supported the growth and proliferation of the cells. There was no negative effect on the growth of cell on the PMMA and its QDs nanocomposite films within the 48 hours of incubation. There is good adhesion and growth in the presence of PMMA-QDs nanocomposite thin films.

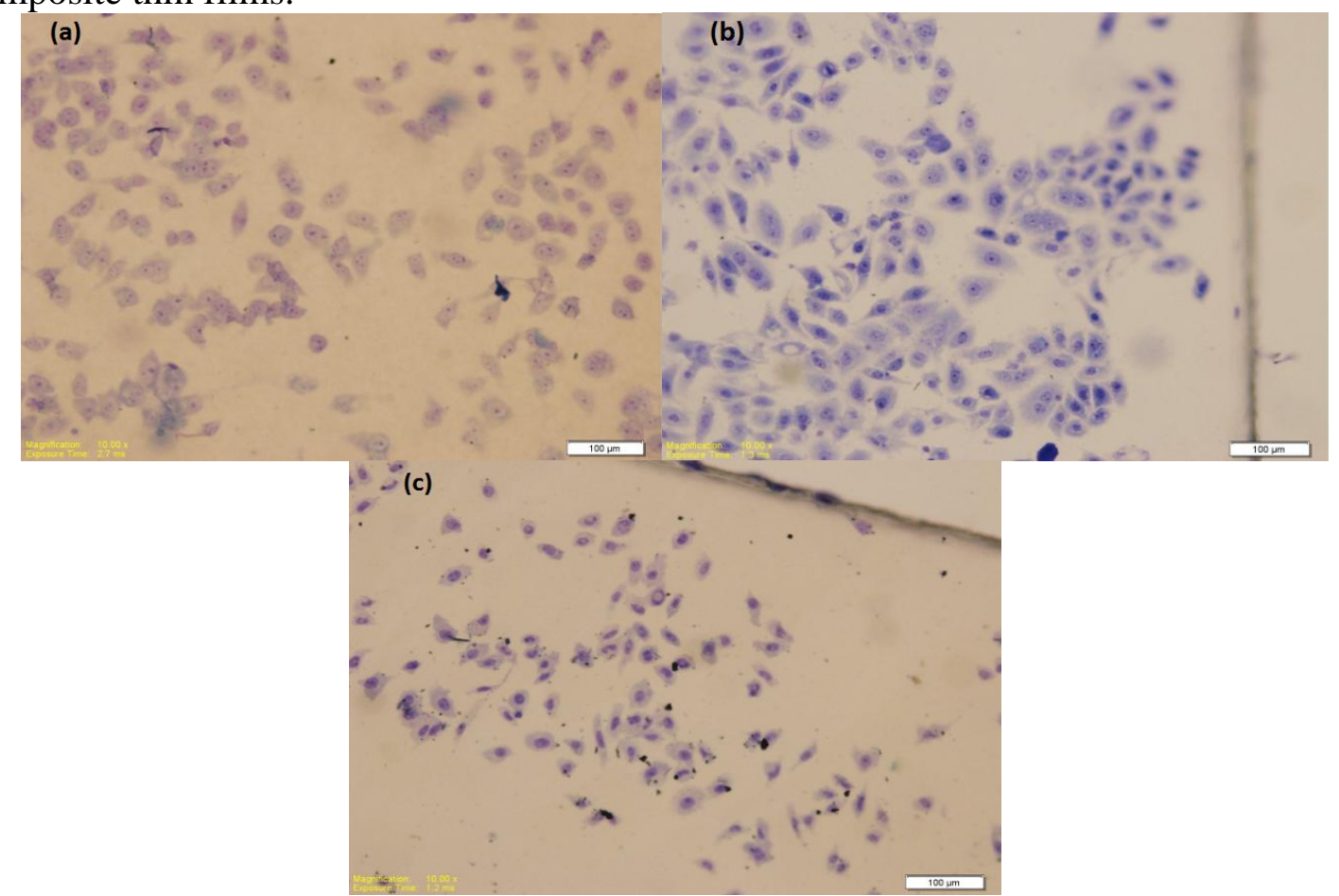

Fig. 5 Cell culture test on (a) a-PMMA-QDs, (b) i-PMMA-QDs, and (c) s-PMMA-QDs 


\section{Acknowledgment}

This research was partially supported by the Welch Foundation (V-0004).

\section{References}

[1] R. Kanemoto, A. Anas, Y. Matsumoto, R. Ueji, T. Itoh, Y. Baba, S. Nakanishi, M. Ishikawa, V. Biju, Relations between dewetting of polymer thin films and phase-separation of encompassed quantum dots, J. Phys. Chem. 112 (2008) 8184-8191.

[2] R. O. Loutfy, D. M. Teegarden, Effect of polymer chain tacticity on the fluorescence of molecular rotors, Macromolecules 16 (1983) 452- 456.

[3] E. J. A. Pope, M. Asami, J. D. Mackenzie, Transparent silica gel PMMA composites, J. Mater. Res. 4 (1989) 1018-1026.

[4] B. Abramoff, L. C. Klein, PMMA impregnated silica gels: synthesis and characterization, Proc. SPIE, Sol-Gel Optics. 1328 (1990) 241-248.

[5] M. C. Nobrega, L. C. F. Gomes, G. P. LaTorre, J. K. West, Microstructure, optical, and dielectric characterization of porous gel silica impregnated with PMMA, Mater. Charact. 40 (1998) 1-5.

[6] P. J. Flory, M. Volkenstein, Statistical mechanics of chain molecules, Biopolymers 1969.

[7] C. S. Reddy, A. Zak, E. Zussman, WS2 nanotubes embedded in PMMA nanofibers as energy absorptive material, J. Mater. Chem. 21(2011) 16086-16093.

[8] R.Q. Frazer, R.T. Byron, P.B. Osborne, K.P. West, PMMA: an essential material in medicine and dentistry, J. Long Term Eff. Med. Implants 15 (2005) 629-639. 